\title{
Electric Propulsion: A Key Enabling Technology for Planetary Exploration*
}

A White Paper for the Planetary Science and Astrobiology Decadal Survey 2023-2032

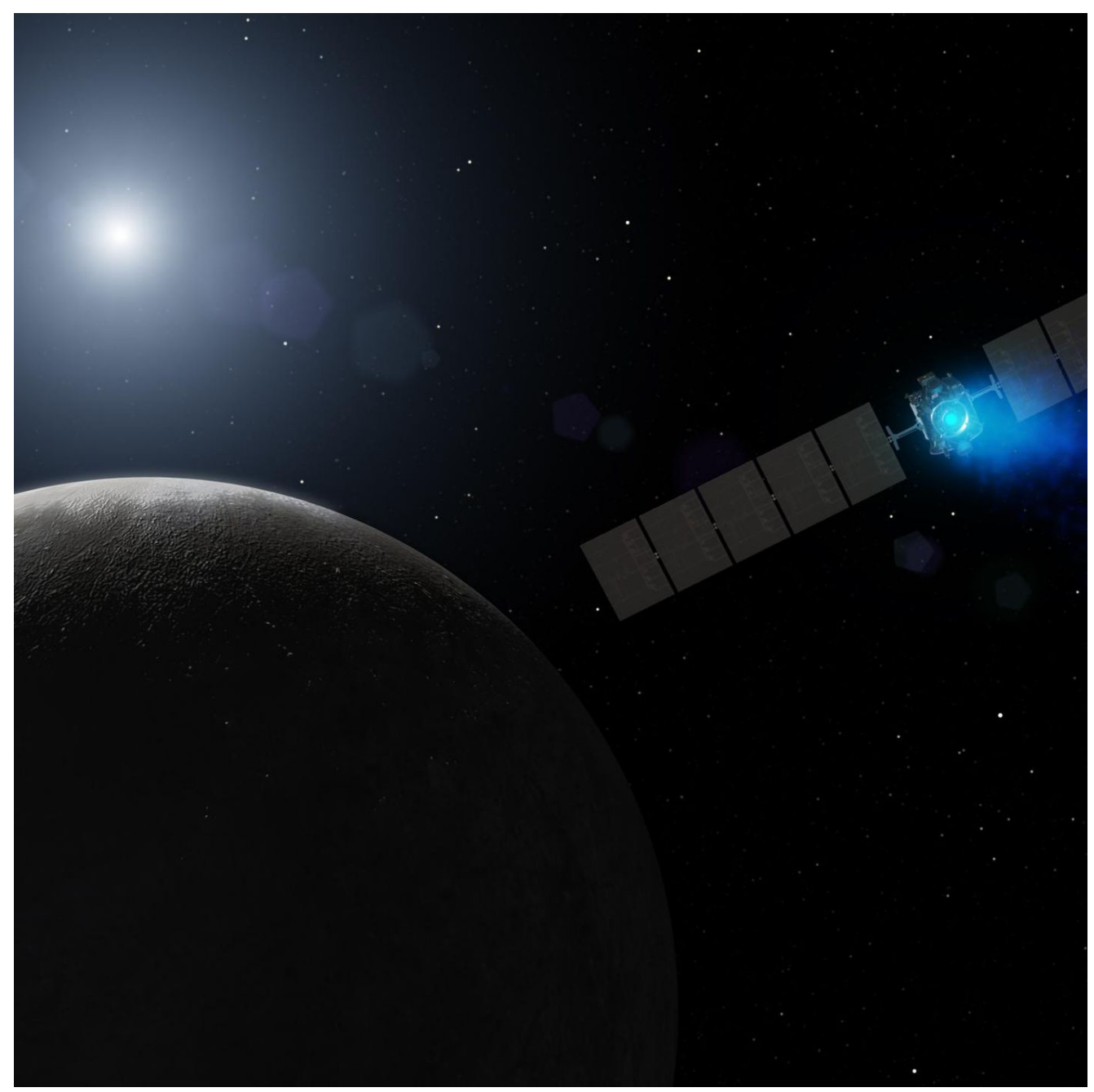

Jay Polk, Jet Propulsion Laboratory, California Institute of Technology

james.e.polk@jpl.nasa.gov, 818-281-1094

\section{George Soulas, NASA Glenn Research Center}

george.c.soulas@,nasa.gov, 216-977-7419

Andy Hoskins, Aerojet Rocketdyne

william.hoskins@rocket.com, 425-945-6362

*The information presented in this white paper about future mission concepts and power systems is predecisional and is provided for planning and discussion purpose only. A portion of this work was carried out at the Jet Propulsion Laboratory, California Institute of Technology, under a contract with the National Aeronautics and Space Administration (80NM0018D0004). 
Abstract. Electric propulsion is now widely accepted by industry, with over 900 operational spacecraft using electric thrusters as of June 30, 2020. Electric propulsion provides dramatic reductions in propellant mass which can enable many demanding mission concepts to meet their scientific and operational goals. It has already proven to be a critical enabling technology on science missions such as Dawn, Hayabusa I and II, and BepiColombo and is the key to making missions in development like Psyche and a potential Mars Sample Return possible. These examples show that electric propulsion can enable the use of smaller launch vehicles, provide mass margins that make missions much more robust, yield much greater flexibility in launch dates, reduce the number of critical events, and, in some cases, reduce trip times by eliminating the need for gravity assists.

Electric propulsion systems developed by industry for commercial spacecraft and NASAdeveloped technologies for higher power and specific impulse support current mission applications, but ensuring that electric propulsion can meet the demands of future missions requires continued investment by NASA. Specific recommendations include support of high specific impulse thruster development, the development of low power systems to enable the use of small spacecraft in low cost missions, adaptation of commercial electric propulsion systems to meet the unique requirements of deep space and development of dual-use systems, and further investments in power electronics, solar array, and nuclear power system technologies.

High-priority science goals dictate more ambitious missions with higher $\Delta V$ s. Solar electric propulsion (SEP) and nuclear electric propulsion (NEP) can enable many of the next generation of planetary exploration mission concepts, which have higher $\Delta$ Vs and more complex spacecraft and mission designs. Planetary missions have become progressively more complex, starting with flybys, then orbiters of the terrestrial planets, followed by Jupiter and Saturn orbiters, and recently by small body missions and ever more complex Mars rovers. Even more difficult proposed missions include sample returns, multi-body rendezvous, ice giant orbiters, landers for outer planet satellites, and further exploration of the Kuiper belt and near-interstellar space.

The white papers submitted for this Decadal Survey show that the planetary science community is highly interested many demanding missions. In addition to the Comet Nucleus Sample Return mission concept which appeared in previous Decadal Surveys and the proposed Mars Sample Return, members of the science community are advocating returning samples from Enceladus [1], Ceres [2], Mercury [3], interstellar objects [4,5], and many small bodies [6]. Multi-body rendezvous missions are also being proposed; for example, orbiter missions to active primordial bodies [7], including main belt comets [8]. A near-term priority is the further exploration of known or candidate ocean worlds [9,10], including a Europa lander [11]; Triton lander [12], a Triton or Pluto hopper mission [13], and a Pluto orbiter or lander [14]. The ice giants Uranus and Neptune have so far only been surveyed during Voyager flybys, so more detailed exploration with orbiter missions is a high priority $[15,16,17]$. There is also considerable interest in further Kuiper belt missions [18,19,20].

While some of these missions could be done with ballistic trajectories, many benefit from or would be enabled by the use of electric propulsion. Because electric thrusters have a specific impulse higher by an order of magnitude or more compared to chemical propulsion, very high $\Delta \mathrm{V}$ missions can be accomplished with much lower propellant mass. This generally means that 
more payload mass can be delivered to a target for a given launch vehicle or that a smaller, less costly launch vehicle can be used, which can enable an otherwise impossible or unaffordable mission. Higher mass margins also give tremendous programmatic flexibility when dealing with the inevitable mass growth during the development of complex missions. Trip times can often be shorter by flying higher energy trajectories or by eliminating the need for time-consuming gravity assists. Other benefits include much greater launch window flexibility and fewer mission critical events, by eliminating impulsive orbit insertion maneuvers, for instance.

Electric propulsion is enabling or enhancing for comet sample return applications [21], sample return missions from Ceres [22] and Enceladus [1]; multi-body rendezvous missions such as a Trojan tour $[23,24]$ or the investigation of multiple MBCs [8,25]; rendezvous with transient ISOs [4]; ice giant orbiters [26,17,27]; and outer planet satellite landers [13]. In fact, advancing electric propulsion technology is one of the key recommendations of the Outer Planets Assessment Group for enabling the exploration of the outer solar system [15].

Electric propulsion has proven to be an enabler for planetary science. The technology demonstration missions Deep Space 1, SMART 1, and Hayabusa I showed that electric propulsion systems were ready for use in science missions. Since then three planetary science missions have been launched with solar electric propulsion--Dawn, Hayabusa II, and BepiColombo--and two more are in development or advanced study phases-Psyche and the Earth Return Orbiter that is part of the planned Mars Sample Return architecture. These represent the first wave of more demanding missions that have benefitted from the use of electric propulsion. The impact of electric propulsion on these missions is discussed in this section.

The Dawn spacecraft rendezvoused with Vesta and Ceres, the two largest main belt asteroids, and returned a wealth of data. NASA launched the $1218 \mathrm{~kg}$ (wet mass) spacecraft in 2007 with a Delta II launch vehicle. The electric propulsion system was powered by a solar array that produced $10 \mathrm{~kW}$ of power at $1 \mathrm{AU}$ and consisted of three NSTAR ion thrusters and two power processing units developed by NASA. The NSTAR ion propulsion subsystem was first flown on the Deep Space 1 technology demonstration mission, which launched in 1998 and flew by the asteroid 9969 Braille in 1999 and comet 19P/Borelly in 2001 [28]. The Dawn electric propulsion system was used first for the heliocentric transfer to Vesta, with a rendezvous in 2011. The system was also used for transfers between a range of science orbits over 14 months, and then the transfer to Ceres. The rendezvous with Ceres occurred in 2014, followed by the 18 month primary mission and two mission extensions. By the end of operations in 2018, the ion propulsion system had delivered over $11.5 \mathrm{~km} / \mathrm{s} \Delta \mathrm{V}$ [29]. This was more than the $\Delta \mathrm{V}$ imparted by the launch vehicle and was achieved with only $416 \mathrm{~kg}$ of xenon propellant, which demonstrates the tremendous advantage electric propulsion provides in reducing propellant mass. A single mission to both asteroids would have been impossible without electric propulsion, and an orbital mission to Vesta or Ceres by itself using chemical propulsion would have been unaffordable in the Discovery program [30].

Hayabusa I, developed and operated by the Japanese Aerospace Exploration Agency (JAXA), was primarily a technology demonstration for future small body sample return missions. Although it encountered a number of technical issues, it successfully returned samples gathered from the asteroid Itokawa. The small spacecraft, with a wet mass of only $510 \mathrm{~kg}$, was launched 
in 2003, rendezvoused with Itokawa in 2005, and returned the samples to Earth in 2010. The 2.6 $\mathrm{kW}$ solar array (at $1 \mathrm{AU}$ ) powered an ion propulsion system consisting of four small microwave ion thrusters. These engines were used for the heliocentric portion of the rendezvous and the earth return phase. A total $\Delta \mathrm{V}$ of $2.2 \mathrm{~km} / \mathrm{s}$ was produced by the electric propulsion system, which consumed only $47 \mathrm{~kg}$ of xenon. This mission was a dramatic demonstration of how electric propulsion can enable sample return missions with small spacecraft. The flexibility of the electric propulsion system also enabled the recovery of the mission after a number of failures that would have crippled it otherwise [31].

The Hayabusa II mission was launched by JAXA in 2014 and rendezvoused with asteroid Ryugu in 2018, where it collected samples and deployed a small lander and two small rovers. The spacecraft left Ryugu in 2019 and will return the samples to Earth late this year. The spacecraft design is based heavily on Hayabusa I, but with more reliable components and more redundancy. These improvements in reliability increased the wet mass to $610 \mathrm{~kg}$. Fifty $\mathrm{kg}$ of propellant will produce a total $\Delta \mathrm{V}$ of about $2 \mathrm{~km} / \mathrm{s}$, similar to Hayabusa I. The spacecraft will have about $30 \mathrm{~kg}$ of xenon remaining after the sample return, and an extended mission to fly by another small body is under consideration [32]. The success of Hayabusa II is the result of JAXA's commitment to developing and demonstrating the technologies for small body sample returns.

The BepiColombo mission is designed to perform a detailed exploration of Mercury with two separate orbiters delivered by a Mercury Transfer Module (MTM) which is propelled by electric thrusters. The MTM and orbiters with a combined $4100 \mathrm{~kg}$ wet mass were launched in 2018 and will be captured at Mercury in late 2025. The solar array produces power levels ranging from 7 $\mathrm{kW}$ at $1.13 \mathrm{AU}$ to $14 \mathrm{~kW}$ at $0.62 \mathrm{AU}$. The electric propulsion system, which employs four T6 ion thrusters manufactured in the UK by Qinetiq, will process $582 \mathrm{~kg}$ of xenon and produce a $\Delta \mathrm{V}$ of $2.7 \mathrm{~km} / \mathrm{s}$. Early mission analyses indicated that a chemical propulsion option was feasible, but was inflexible to mass increases, so the electric propulsion option was baselined. Had the chemical option been chosen at that time, it would have resulted in a brittle design that would not have survived subsequent mass growth, and the science payload would have been drastically descoped [33]. This is an example of how the greater margins afforded by electric propulsion can make complex, demanding missions extremely robust. This advantage was also critical to the success of the Dawn mission. For example, the SEP system afforded margins that allowed the Dawn spacecraft to arrive at Vesta months early, yielding greater schedule reserve and more observations at the target [30].

The Psyche mission, which is currently in development, will use an electric propulsion system developed for commercial satellites to deliver its science payload to the metal asteroid Psyche. It is scheduled to launch in 2022 and rendezvous with Psyche in 2026, where it will begin its 21 month science phase. The $2800 \mathrm{~kg}$ spacecraft is a hybrid of the structure, power, and electric propulsion subsystems from Maxar's 1300 series product line of commercial communications satellites and JPL's heritage deep space avionics. A $20 \mathrm{~kW}$ solar array provides power for the propulsion system, which uses four SPT-140 Hall thrusters. A propellant load of $1052 \mathrm{~kg}$ of xenon will yield a $\Delta \mathrm{V}$ of $5.7 \mathrm{~km} / \mathrm{s}$. In this case, the use of electric propulsion enables the exploration of Psyche using a medium-class launch vehicle, which makes it possible within the Discovery cost cap [34]. 
The Earth Return Orbiter (ERO) for the proposed Mars Sample Return mission will also employ electric propulsion as part of a complex architecture in the planning phase. NASA and ESA are studying a concept that would include the Mars 2020 Perseverance rover to collect and cache samples, a US-supplied spacecraft to deliver a European rover to fetch the cached samples and an ascent stage to launch them in an Orbiting Sample (OS) container. The ERO supplied by ESA and associated US subsystems would capture the OS and return it to Earth. The ERO could launch as early as 2026 with a five year round-trip mission. Mission analyses have shown that all-electric propulsion options are very mass efficient, but can't meet the mission timeline. Allchemical propulsion options require a heavy lift launch vehicle, staging, and long aero-braking phases to be feasible, as well as suffering from significant programmatic risk related to mass margins. The current baseline, a hybrid chemical/electric system, has been identified as the best option [35]. A $41 \mathrm{~kW}$ (1 AU) solar array would power four RIT-2X ion engines produced by the Ariane Group in a SEP system that would be used for the heliocentric transfer to Mars. A bipropellant system would insert the spacecraft into a highly elliptical orbit around Mars. The SEP system would be used for a subsequent spiral down to the OS rendezvous orbit, then spiral out and return the samples to Earth. The hybrid system represents a compromise that benefits from the mass efficiency of electric propulsion to provide about $10 \mathrm{~km} / \mathrm{s} \Delta \mathrm{V}$ in the transfers, but avoids the long time required for a low-thrust capture at Mars by using a high thrust chemical propulsion module [35].

These examples demonstrate that many demanding missions can be enhanced or enabled by electric propulsion, with system power levels ranging from several $\mathrm{kW}$ to over $40 \mathrm{~kW}$ and individual thruster power levels of a few hundred watts up to $8.5 \mathrm{~kW}$. They reveal a trend to higher power systems for ambitious orbiter missions that leverage advances in solar array power technology driven by commercial as well as NASA needs. However, the Hayabusa missions show the promise offered by smaller spacecraft for lower cost missions that can still deliver exciting, high quality science.

The state-of-the-art technology only spans part of NASA's needs. Electric propulsion offers many advantages for planetary exploration, but only part of this vision can be realized with existing electric propulsion technology. The current options for near-term missions are summarized briefly in this section.

Many of the missions listed above relied on high specific impulse ion engines. The NSTAR thrusters flown on Dawn, for example, produced an Isp of $3100 \mathrm{~s}$ at a peak power of $2.5 \mathrm{~kW}$. Although these thrusters are no longer available from the manufacturer, NASA's Evolutionary Xenon Thruster (NEXT) exceeds NSTAR's capabilities. NEXT is a throttleable engine developed by NASA for planetary and commercial applications and manufactured by Aerojet Rocketdyne. It produces up to $4200 \mathrm{~s}$ specific impulse at power levels of 0.64 to $7.33 \mathrm{~kW}$. A NEXT system consisting of a single thruster and power processing unit is being integrated onto the Double Asteroid Redirection Test (DART) spacecraft and will be operated as a technology demonstration [36]. NEXT is enabling for applications like comet sample return missions (and, in fact, was baselined on the proposed Comet Astrobiology Exploration Sample Return (CAESAR) mission concept, which completed a Step 2 study in the last Discovery round but was not selected to go into development), as well as demanding outer planet Flagship missions. 
NASA is also developing higher power Hall thrusters for deep space applications. The Advanced Electric Propulsion System (AEPS), which consists of a state-of-the-art Hall thruster capable of operating at power levels ranging from 7 to $13.5 \mathrm{~kW}$ and specific impulses as high as $2700 \mathrm{~s}$, is under development for the Power and Propulsion Element (PPE) of the lunar Gateway [37]. The PPE is being built by Maxar and will fly two AEPS thrusters to be built by Aerojet Rocketdyne (AR), four $6 \mathrm{~kW}$ Hall thrusters to be built by Busek, and a $60 \mathrm{~kW}$ solar array [38]. The technologies demonstrated in this human exploration mission could also benefit robotic planetary applications that require higher power, such as solar-powered outer planet missions.

Electric propulsion systems developed for commercial communications satellites have high heritage and can provide an affordable option for cost-constrained competed missions; however, there are currently only a few qualified systems available. The SPT-140 Hall thrusters that will be used on Psyche have flown on five Maxar spacecraft to date [39]. They operate at power levels of 3.0 to $4.5 \mathrm{~kW}$ and specific impulses up to $2000 \mathrm{~s}$. The AR XR-5 Hall thruster system operates at up to $3.0 \mathrm{~kW} /$ thruster and $1860 \mathrm{~s}$ specific impulse and has flown on 10 Lockheed Martin and Northrup Grumman geosynchronous spacecraft to date. [40]. AR is developing a second generation 3-7 kW Hall thruster system expected to be ready for flight as early as 2025 . The AR built MR-510 arcjet system, operating between 1.6 to $2.2 \mathrm{~kW}$ per thruster, has flown on more than 55 spacecraft and is able to boost the performance of hydrazine from $220 \mathrm{~s}$ to a mission average $585 \mathrm{~s}$ [41]. The $6 \mathrm{~kW}$ Hall thruster system that will fly on the PPE is currently under development by Maxar and Busek for future commercial satellites.

Trajectory analyses for Discovery mission concept studies and full proposals have shown that these performance parameters are of interest for many Discovery-class mission applications, for example, the Psyche mission. However, there are some important differences between commercial requirements and planetary science mission needs. Commercial systems are often limited to just a few operating points (for orbit-raising and stationkeeping, for example) and do not need to be highly throttleable. Lifetime requirements are often less demanding for commercial systems. On planetary spacecraft the solar array voltage can often vary significantly with solar range due to temperature and illumination intensity variations. Commercial power processing units (PPUs) for electric propulsion systems are generally not designed to accommodate a large range of input voltages. These differences can be addressed with additional testing, modeling, and minor design changes. For example, the Psyche project conducted tests to show that the SPT-140 thruster had sufficient throttling capability and made minor modifications to the power processing unit to accommodate additional throttling. Lifetime at throttled conditions was addressed with modeling and testing [42]. As commercial electric propulsion systems evolve, the need for these adaptions for use in deep space will continue.

Recommendations to NASA. Electric propulsion is an enabling technology for many ambitious missions envisioned in this Decadal Survey, but NASA must continue investing in the advancement of the technology. We recommend the following specific areas of investment to address future mission needs.

- Support the continued development of high Isp propulsion options. NASA should continue the infusion of the NEXT ion propulsion system into planetary science missions and extend the Isp range of Hall thruster technology. 
- Support the development of lower power thrusters for small spacecraft. The capabilities of miniaturized instruments and spacecraft subsystems are increasing rapidly and could enable a new class of affordable planetary missions. However, there are few options for high delta-V propulsion on small spacecraft. NASA should support the development of highly reliable, long life, sub-kW Hall and ion engine systems like those on the Hayabusa spacecraft.

- Support the adaptation of commercial systems for deep space applications. Commercial systems provide an affordable option for cost-constrained Discovery missions. Deep space applications may require demonstrating thruster operation over an expanded throttle range, modifications to propellant feed systems or power processing units to support throttling, delta qualification for minor differences in environmental requirements, and testing and modeling to support longer life requirements or life assessment at throttled conditions.

- Support modeling and simulation of thruster lifetime and development of accelerated wear test methods. Because electric thrusters require very long burn times (tens of thousands of hours), life qualification can be costly and technically difficult. Understanding failure modes and incorporating physics-based modeling in the qualification process as well as developing methods for accelerated testing are critical for managing risk and qualification cost.

- Support the development of advanced, flexible power processing units. Power processing units are often the most expensive and complex part of an electric propulsion system. The infusion of new power processing technologies can improve efficiency, reduce mass, and provide better peak power tracking (as outlined in the white paper by Carr, et al. [43]). Because power processing units are expensive and time-consuming to develop, NASA should continue the development of a system with the flexibility to run multiple types of thrusters [44].

- Support advanced solar and nuclear power system development. Mission performance with electric propulsion is sensitive to power level and mass of the power system. Schwartz, et al. make recommendations for increasing solar array power and performance [45], while Casani, et al. advocate fission power systems to enable ambitious missions to the outer planets [46].

\section{References}

[1] Neveu, M, et al., 2020, "Returning Samples from Enceladus for Life Detection," Decadal Survey White Paper

[2] Castillo-Rogez, J., et al., 2020 "Science Motivations for the Future Exploration of Ceres," Decadal Survey White Paper

[3] Vander Kaaden, K.E., et al., 2020, "Mercury sample return to revolutionize our understanding of the solar system," Decadal Survey White Paper

[4] Hein, A.M., et al., 2020, "Interstellar Now! Missions to and Sample Returns from Nearby Interstellar Objects," Decadal Survey White Paper

[5] Meech, K.J., et al., 2020, "In-Situ Exploration of Objects on Oort Cloud Comet Orbits: OCCs, Manxes, ISOs," Decadal Survey White Paper

[6] Jacobson, S., et al., 2020, "Small Bodies Tell the Story of the Solar System: A Scientific Rationale for a Multi-Target Small Body Sample Return Program," Decadal Survey White Paper

[7] Harris, W., et al., 2020, "Active Primordial Bodies: Exploration of the primordial composition of icerich planetesimals and early-stage evolution in the outer solar system," Decadal Survey White Paper

[8] Meech, K.J., et al., 2020, "Main Belt Comets as Clues to the Distribution of Water in the Early Solar System," Decadal Survey White Paper

[9] Hendrix, A.R., et al., 2020, "Ocean Worlds: A Roadmap for Science and Exploration," Decadal Survey White Paper

[10] Howell, S.M., et al., 2020, "Ocean Worlds Exploration and the Search for Life," Decadal Survey White Paper 
[11] Phillips, C.B., et al., 2020, “An Exploration Strategy for Europa,” Decadal Survey White Paper [12] Hansen, C.J., "Triton: Fascinating Moon, Likely Ocean World, Compelling Destination!” Decadal Survey White Paper

[13] Izenberg, N., et al., "Hopper Missions to Triton and Pluto using a Vehicle with In-Situ Refueling," Decadal Survey White Paper

[14] Robbins, S.J., Pluto System Follow-On Missions: Background, Rationale, and New Mission Recommendations," Decadal Survey White Paper

[15] Moore, J., et al., 2020, "Exploration Strategy for the Outer Planets 2023-2032: Goals and Priorities," Decadal Survey White Paper

[16] Blanc, M., et al., 2020, "Science Goals and Mission Objectives for the Future Exploration of Ice Giants Systems: a Horizon 2061 Perspective," Decadal Survey White Paper

[17] Balint, T.S., et al., "Uranus System Exploration Under the New Frontiers Mission Class," Decadal Survey White Paper

[18] Brisset, J., et al., 2020, "Understanding the Formation and Evolution of the Kuiper Belt by Exploring the Haumea System," Decadal Survey White Paper

[19] Holler, B.J., "Prospects for Future Exploration of the Trans-Neptunian Region,” Decadal Survey White Paper

[20] Runyon, K.D., "Comparative Planetology of Kuiper Belt Dwarf Planets Enabled by the Near-Term Interstellar Probe," Decadal Survey White Paper

[21] Veverka, J., et al., 2008, Comet Surface Sample Mission Study, SDO-11998

[22] Castillo-Rogez, J. and Brophy, J.R., 2020, “Exploration of Ceres' Habitability,” Decadal Survey Mission Concept Study

[23] Saikia, S.J., et al., 2014, $11^{\text {th }}$ International Planetary Probe Workshop, 8076

[24] Oh, D., et al., 2005, $29^{\text {th }}$ International Electric Propulsion Conference, IEPC-2005-181

[25] Meech, K.J., 2015, Astrobiology Science Conference 2015, 7433

[26] Conversano, R., et al., 2019, IEEE Aerospace Conference, doi: 10.1109/AERO.2019.8741678

[27] Landau, D., et al., 2010, Advances in the Astronautical Sciences, 125:2093-2112

[28] Brophy, J.R., 2002, Review of Scientific Instruments, 73, 1071, doi: 10.1063/1.1432470

[29] Garner, C.E. and Rayman, M.D., 2018, AIAA Propulsion and Energy Forum, doi: 10.2514/6.20184641

[30] Russel, C.T. and Raymond, C.A., 2011, Space Sci. Rev. 163:3-23, doi: 10.1007/s11214-011-9836-2

[31] Nishiyama, K., et al., 2012, Trans. JSASS Aerospace Tech. Japan, 10:1-8, doi: 10.2322/tastj.10.tb_1

[32] Nishiyama, K., et al., 2015, AIAA Propulsion and Energy Forum, doi: 10.2514/6.2015-3718

[33] Sutherland, O., et al., 2019, 38 ${ }^{\text {th }}$ International Electric Propulsion Conference, IEPC-2019-824

[34] Oh, D., et al., 2016, AIAA Propulsion and Energy Forum, doi: 10.2514/6.2016-4541

[35] Sutherland, O., et al., 2019, $38^{\text {th }}$ International Electric Propulsion Conference, IEPC-2019-A-927

[36] Fisher, J., et al., 2020, AIAA Propulsion and Energy Forum, doi: 10.2514/6.2020-3604

[37] Zubair, J., et al., 2020, AIAA Propulsion and Energy Forum, doi: 10.2514/6.2020-3628

[38] Herman, D.A., et al., 2019, International Electric Propulsion Conference, IEPC-2019-651

[39] Johnson, I., et al., 2020 AIAA Propulsion and Energy Forum, AIAA 2020-3606

[40] Glogowski, M.J., et al. 2018, AIAA Propulsion and Energy Forum, AIAA 2018-4719

[41] Zube, D.M.; et al., 2017, International Electric Propulsion Conference, IEPC-2017-305

[42] Kerl, T., et al., 2020, AIAA Propulsion and Energy Forum, doi: 10.2514/6.2020-3605

[43] Carr, G., et al., 2020, "Power Electronic Technologies for Planetary Science and Astrobiology Missions," Decadal Survey White Paper.

[44] Kamhawi, H., et al., 2016, AIAA Propulsion and Energy Forum, doi: 10.2514/6.2016-4943

[45] Schwartz, J.A., et al., 2020, "Solar Array Technologies for Planetary Science and Astrobiology Missions," Decadal Survey White Paper.

[46] Casani, J.R., et al., "Enabling a New Generation of Outer Solar System Missions: Engineering Design Studies for Nuclear Electric Propulsion,” Decadal Survey White Paper 